\title{
EchoGéo
}

35 | 2016

Les découpages territoriaux en Europe

\section{La réforme régionale en France : une occasion manquée?}

\author{
Élisabeth Bonnet-Pineau
}

\section{(2) OpenEdition \\ 12 Journals}

Édition électronique

URL : https://journals.openedition.org/echogeo/14506

DOI : 10.4000/echogeo. 14506

ISSN : 1963-1197

\section{Éditeur}

Pôle de recherche pour l'organisation et la diffusion de l'information géographique (CNRS UMR 8586)

Référence électronique

Élisabeth Bonnet-Pineau, «La réforme régionale en France : une occasion manquée? 》, EchoGéo [En ligne], 35 | 2016, mis en ligne le 19 avril 2016, consulté le 11 août 2021. URL : http:// journals.openedition.org/echogeo/14506; DOI : https://doi.org/10.4000/echogeo.14506

Ce document a été généré automatiquement le 11 août 2021.

EchoGéo est mis à disposition selon les termes de la licence Creative Commons Attribution - Pas d'Utilisation Commerciale - Pas de Modification 4.0 International (CC BY-NC-ND) 


\title{
La réforme régionale en France : une occasion manquée?
}

\author{
Élisabeth Bonnet-Pineau
}

1 La loi du 16 janvier 2015 relative à la délimitation des régions modifie la carte des régions françaises. À compter du $1^{\mathrm{er}}$ janvier 2016, l'article $1^{\mathrm{er}}$ de la loi du 16 janvier 2015 substitue 13 régions aux 22 régions métropolitaines existantes.

Illustration 1 - les anciennes et les nouvelles régions françaises

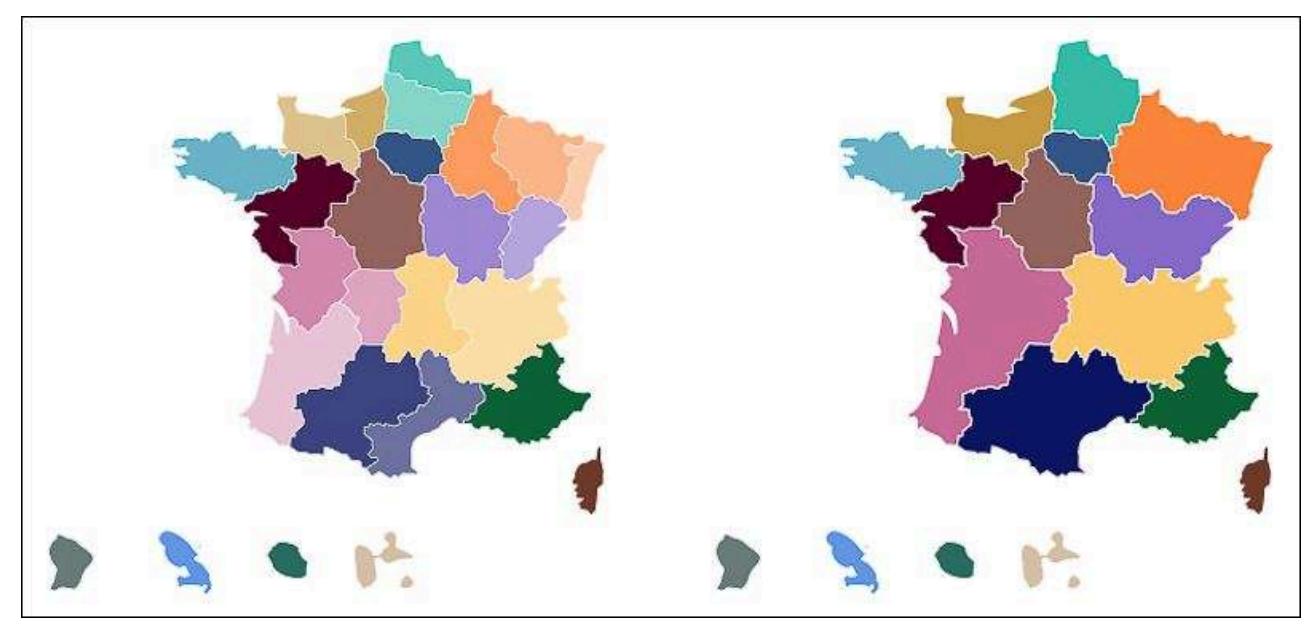

Source : Ministère de l'Intérieur.

2 L'objectif assigné à la réforme est triple : elle vise à « doter les régions françaises d'une taille critique qui leur permette d'exercer à la bonne échelle les compétences stratégiques qui leur sont attribuées, de rivaliser avec les collectivités comparables en Europe et de réaliser des gains d'efficience » (projet de loi nº36 (2013-2014) portant nouvelle organisation territoriale de la République). 


\section{Une réforme nécessaire}

3 - La question du périmètre tout comme celle de la taille des régions est récurrente. Dès 1972, lors de la mise en place des établissements publics régionaux, leur périmètre était déjà jugé trop restreint au regard de l'exercice des compétences tant en matière de développement économique que pour les réseaux de transport. D'ailleurs nombre de rapports notamment à partir de 2004 et surtout de 2008 (rapport Warsmann, rapport Belot, rapport Balladur, rapport Krattinger, sans oublier le rapport Attali) ont préconisé une réduction du nombre des régions et recommandé une réduction du nombre des collectivités exerçant à un même échelon.

4 La délimitation de grandes régions vues d'Europe, avec l'Allemagne en ligne de mire, consiste à atteindre un optimum régional, une taille idéale qui n'existe pas faute de corrélation entre taille, développement économique et richesse. En outre, les structures territoriales des États européens présentent une extrême diversité résultant d'un héritage historique et de choix institutionnels effectués par les États membres.

5 La carte n'est que partiellement redessinée. Des élus de grande notoriété ont été à la manœuvre. Plusieurs fiefs politiques ont résisté. La Bretagne a été épargnée mais l'ambition de maintenir la gauche en Languedoc-Roussillon a abouti à la création d'une région XXL (Midi-Pyrénées + Languedoc-Roussillon) refusée par la Commission spéciale du Sénat. Par contre, la volonté de la fédération socialiste du Nord de conserver la région Nord-Pas-de-Calais n'a pas réussi à empêcher la fusion avec la Picardie.

6 - La question des compétences est un véritable serpent de mer. Le découpage de la France en collectivités territoriales régionales est inédit. Prévalait jusque-là un débat politique uniquement orienté sur l'organisation décentralisée de l'État. Il revient au législateur de déterminer les compétences entre département et région. Elle n'avait alors qu'un statut législatif à la différence des communes et des départements. Les départements ont d'ailleurs perçu les régions comme des concurrentes. La méfiance resta de mise. Tard venue dans le paysage des collectivités territoriales, la région se voit reconnaître en 1982 la preuve de son aptitude à innover, planifier et à coordonner les investissements publics. La région est en somme née dans une " relative discrétion » en demeurant dans le cadre de l'établissement public territorial qui lui-même était le cadre de la circonscription administrative.

7 - La recherche de la clarification des compétences est restée lettre morte ou quasi. La dualité département/région rend toute clarification compliquée. Dans les rapports comme dans les débats au parlement, il apparaît qu'aucune attribution de compétences n'est incontestable, que tout peut être discuté, que rien ne saurait s'imposer avec évidence. La complexité de la redistribution des compétences n'implique pas les seules collectivités territoriales mais aussi l'État. La réticence de l'État, voire son impuissance, à engager une nouvelle étape de décentralisation persiste, ce qui a fait dire à Alain Rousset que la gestion est restée " colbertiste».

8 - Rien de tel à l'échelon de la commune. Depuis la Révolution en effet, la solution choisie est celle du dédoublement fonctionnel, le maire étant d'abord un agent de la commune mais aussi un agent de l'État dans certains cas.

9 Mailles locales, les communes sont les premières accusées d'obsolescence et d'inadaptation à la réalité des modes de vie et des espaces de vie des Français. L'émiettement communal, souvent présenté comme "une exception française » en 
Europe (ce qui laisse supposer qu'il existe une norme), représente $40 \%$ du total des communes européennes (Giblin, 2015). La trame très morcelée des communes regroupe la population dans de petites unités. La persistance d'un tel maillage est étroitement liée à l'histoire rurale de la France et au maintien de la paysannerie dans les campagnes jusqu'au milieu du XXe siècle.

10 C'est aussi durant la période révolutionnaire que les communes ont été dotées d'un cadre de vie politique et qu'elles se virent attribuer la fonction d'établir l'État civil.

11 L'attachement des Français aux mailles locales notamment aux communes perçues comme des échelons de proximité demeure. Tout comme l'ancrage territorial des élus locaux aux différents échelons permet d'assurer le relais entre le local et le national. Cette «classe politique» française numériquement forte exerce un réel rôle intégrateur et permet aux habitants de la « France d'en bas » de se sentir représentés.

12 - La réforme entend faire de l'intercommunalité le principal acteur local en portant le seuil de constitution d'une communauté de communes de 5000 à 20000 habitants. Seuil qui dans les campagnes peu peuplées voire dépeuplées et dans les zones de montagne est jugé trop large. Le passage de 5000 à 20000 habitants apparaît précipité même si le principal gisement susceptible de favoriser des économies budgétaires se situe dans le renforcement des intercommunalités.

13 Si le phénomène d'intercommunalité est ancien, les lois du 6 février 1992 et du 12 juillet 1999 l'ont renforcé. L'intercommunalité se réalise dans les EPCI ${ }^{1}$ (Établissements publics de coopération intercommunale) à fiscalité propre qui assument la gestion des territoires.

14 Les intercommunalités peinent cependant à s'imposer comme cadre de référence. L'absence d'élection au suffrage universel direct entretient le "déficit de légitimité ». La France n'offre pas un cas isolé. La question supra-communale se pose dans la plupart des pays européens d'une manière ou d'une autre, quand bien même une vague de rationalisation des maillages communaux a eu lieu.

15 Les échelons communal, départemental et régional ont fortement structuré le système d'administration territoriale de la France. La forte inertie de ces trois maillages a d'autant plus résisté que «la décentralisation n'a pas réussi à organiser l'interterritorialité » (Vanier, 2008).

16 Autrement dit, si depuis la Révolution, le système d'administration territoriale a été marqué par le principe d'uniformité considéré comme gage d'unité, il serait possible aujourd'hui d'y renoncer en procédant à une déconcentration des pouvoirs de l'État.

17 - La question des gains d'efficience. Les tenants et défenseurs du nouveau découpage régional ont fait valoir l'argument d'économies générées du fait même de la réduction du nombre de régions. André Vallini, le secrétaire d'Etat chargé de la Réforme territoriale a avancé le chiffre de 12 milliards d'économies en mai 2014 en se fondant sur une réduction de 5 à $10 \%$ des dépenses totales des collectivités, chiffres qu'il a revus à la baisse en juin 2014 en précisant que de telles économies ne seraient réalisées que d'ici à 10 ans pour arriver à une dizaine de milliards d'euros... De telles annonces ont suscité bien des réactions et traduisent des faiblesses de la méthode. Les estimations d'André Vallini portaient, il est vrai, sur l'ensemble de la réforme territoriale, principalement sur le projet de loi «NOTRe» (Nouvelle Organisation Territoriale Régionale) qui prévoyait des mutualisations et des transferts de compétences entre échelons, des départements vers les régions. 
18 Aucune évaluation de ces gains d'efficience n'a été faite à l'exception de la direction générale des collectivités locales qui a dressé un inventaire des gisements d'économies. Si économies il y a, elles résulteront des décisions des collectivités elles-mêmes et des mutualisations qu'elles parviendront à mettre en œuvre. Les économies seront faites sur les budgets de fonctionnement des collectivités aux dépens des investissements.

19 En réalité, grand est le risque que la fusion des régions ne se traduise par un surcoût, ne serait-ce que par l'alignement des conditions salariales des agents et de leurs statuts et des coûts directs liés aux déménagements, à la mise en place d'outils informatiques communs, etc. Selon le géographe Gérard-François Dumont, « l'expérience montre que les coûts croissent avec la taille ${ }^{2}$ ».

20 Pèse un facteur d'incertitude sur l'allocation des ressources et de la fiscalité puisque la réforme du mode de calcul de la dotation globale de fonctionnement (DGF) a été inscrite dans le projet de loi de finances pour 2016. La réforme actuelle fait table rase des modalités de répartition et se cale sur la réalité des territoires et de leur population. La réforme de la DGF aurait pu faire l'objet d'une loi spécifique ultérieure. Le gouvernement se dit prêt à accepter la clause de revoyure afin de rétablir notamment les situations des EPCI. Les communautés d'agglomération et les communautés de communes sont les principales gagnantes.

Le redressement des comptes publics ne se fera certainement pas grâce à la fusion des régions.

Quant au renforcement de la puissance économique des nouvelles régions dont la taille est supposée être un gage de dynamisme de développement, la prudence est de mise. La taille n'est pas un critère de réussite. Prenons l'exemple des trois régions du Nord-Est qui sont promises à un avenir commun dans le cadre d'une nouvelle entité régionale de grande taille.

23 L'Alsace est une région dont le dynamisme économique s'accommode fort bien de sa petite taille. Fusionner l'Alsace et la Lorraine, traditionnellement dissociées par leur histoire et leur inclination plus que par la coupure des Vosges n'apporte aucune garantie de dynamisme de développement. La fusion n'aura pas des effets nécessairement positifs.

24 Si la Lorraine a été « un laboratoire d'expériences de l'Europe » en se tournant vers le Luxembourg et en profitant de la proximité de la Sarre et de la Wallonie, l'Alsace regarde vers l'Est, vers le monde rhénan et Strasbourg, promue métropole européenne, bénéficie d'un véritable hinterland européen.

25 Accoler la Champagne-Ardenne à l'ensemble Alsace-Lorraine après l'avoir ballotée entre Picardie et Lorraine telle une "variable d'ajustement" révèle et confirme l'absence de vision stratégique et une volonté d'ignorer la réalité du tropisme rhénan de l'Alsace au risque de créer un axe de fragilisation dans l'espace lorrain intermédiaire.

\section{Une réforme inachevée}

26 - Une réforme menée au forceps. Alors que les délimitations des régions françaises et même les noms étaient restés inchangés (ou presque, seule la Corse s'autonomisant) depuis l'introduction des «régions de programme» en 1956, l'accélération du calendrier mérite d'être soulignée. Entre le discours prononcé par le Président de la 
République annonçant le redécoupage régional et la promulgation de la loi délimitant les régions, une année s'est écoulée. Et la nouvelle loi est entrée en vigueur le $1^{\text {er }}$ janvier 2016.

La survenance soudaine de la refonte de la carte régionale et la rapidité des effets ont créé la surprise.

En mettant en avant la taille des régions depuis les années 2000 en lien avec les élargissements successifs, la thèse des grandes régions s'est imposée en faisant valoir des économies d'échelle importantes. L'effet de taille s'est imposé comme un postulat non discutable devant permettre d'accroître la compétitivité des structures administratives et leur complémentarité avec les aires métropolitaines qui les structurent.

Tableau 1 - La réforme territoriales en chiffres

\begin{tabular}{|c|c|c|c|c|}
\hline Régions * & $\begin{array}{l}\text { Population } \\
\text { municipale (en } \\
\text { millions d'hab.) }\end{array}$ & $\begin{array}{l}\text { Dépenses } \\
\text { réelles totales } \\
(2012)\end{array}$ & $\begin{array}{l}\text { Dépenses totales } \\
\text { par habitant (en } \\
€)\end{array}$ & $\begin{array}{l}\text { Economies } \quad \text { réalisées } \\
\text { dans } \\
\text { retenue (millions } € \text { ) }\end{array}$ \\
\hline Limousin & 0,7 & 406 & 548 & 104 \\
\hline $\begin{array}{l}\text { Franche- } \\
\text { Comté }\end{array}$ & 1,2 & 485 & 413 & 6 \\
\hline $\begin{array}{l}\text { Champagne- } \\
\text { Ardenne }\end{array}$ & 1,3 & 601 & 450 & 56 \\
\hline Auvergne & 1,4 & 641 & 475 & 90 \\
\hline $\begin{array}{l}\text { Basse- } \\
\text { Normandie }\end{array}$ & 1,5 & 624 & 423 & 22 \\
\hline Bourgogne & 1,6 & 722 & 440 & 52 \\
\hline Picardie & 1,9 & 822 & 429 & 39 \\
\hline $\begin{array}{l}\text { Languedoc- } \\
\text { Roussillon }\end{array}$ & 2,7 & 1114 & 417 & 25 \\
\hline & & & & 393 \\
\hline
\end{tabular}

*Concernées par la réforme et ayant un niveau de dépenses par habitant supérieur à la moyenne Source : La réforme territoriale, rapport du Sénat du 26/06/2014, p 27.

Les économies d'échelle attendues apparaissent marginales. Les régions les moins peuplées ont en moyenne un niveau de dépenses plus élevé par habitant. La fusion des régions laisse espérer un alignement à la baisse des niveaux de dépenses par habitant. Mais la vérité qui prévaut ici ne s'applique pas nécessairement ailleurs. D’autres critères peuvent favoriser la fusion des régions. Ainsi la fusion des régions BourgogneFranche-Comté est justifiée par la coopération existant dans l'enseignement supérieur. 
Mais alors qu'existe un rapprochement entre les universités de Bretagne et des Pays de la Loire, la fusion des deux régions n'a pas été adoptée.

31 Manque de méthode ou plutôt fragilité des fondements de redécoupage... Les préconisations d'une mission parlementaire qui envisageaient la possibilité de regroupement volontaire ou de modification des limites territoriales des régions, sur proposition concordante des assemblées délibérantes des territoires concernés statuant à la majorité et ratifiée par référendum à l'issue d'un large débat n'a pas eu lieu. La refonte de la carte des régions de France relève de « l'art de la découpe ».

D'autres fondements de découpage auraient pu être introduits en s'appuyant sur les métropoles et leur attractivité, sur les mises en réseaux ou sur les bassins de vie.

- La sauvegarde des principes classiques de l'organisation territoriale française a été rendue possible grâce à la compatibilité avec la constitution administrative de la France. La refonte des limites des régions est un procédé soluble dans le schéma général d'organisation territoriale française. Demeurent « des régions ordinaires » dont le statut peut être modifié par la loi d'une législature à l'autre. Le Conseil Constitutionnel a rappelé que le principe de libre administration des collectivités territoriales «n'impose pas la consultation des Collectivités territoriales préalablement au dépôt d'un projet ou à l'adoption d'une loi modifiant leurs délimitations territoriales".

La loi du 16 janvier 2015 s'inscrit dans ce schéma par ses dispositions relatives à la fixation du chef-lieu des nouvelles régions issues de regroupement, prérogative laissée au pouvoir réglementaire national, les collectivités visées n'étant saisies que pour simple avis.

La recomposition de la carte régionale s'est faite par l'addition de régions actuelles entières sans modification des départements qui les composent.

Le secrétaire d'État à la Réforme de l'État et à la simplification avait annoncé que la réorganisation territoriale de l'État s'accompagnera de deux réformes essentielles : la revue des missions et la déconcentration des pouvoirs... Les préfets des grandes régions auront un rôle beaucoup plus stratégique que les actuels préfets de région, les préfets de département étant plutôt en charge de la déclinaison opérationnelle des politiques publiques. Depuis 2010, la réforme de l'administration territoriale de l'État s'inscrivait dans le cadre de la révision générale des politiques publiques devenue ensuite la modernisation de l'action publique. La RéATE se voulait une réforme globale de l'État sur le territoire et confirmait le niveau régional comme le niveau de droit commun du pilotage des politiques publiques.

En instituant le $1^{\mathrm{er}}$ janvier 2010 des directions départementales interministérielles (DDI), l'administration départementale a été restructurée - à l'exception de l'île-deFrance, de la Corse et de l'Outre-Mer -, conservant au préfet quelques compétences sensibles pour lesquelles il reste subordonné directement au gouvernement (pouvoir de police, droit des étrangers).

Début mars 2015, Thierry Mandon a assuré en Conseil des Ministres qu'une des plus puissantes réformes de l'État pour ce qui relève de l'Etat territorial avait été engagée ; il annonçait l'arrêt de mort de la RéATE. réorganisation des services de l'État en se doublant d'une revue des missions de l'État. 
La redistribution des services de l'État dans les régions s'est accompagnée depuis 1992 de la recherche de cohérence et d'unité d'action de l'État. Apparaît et se confirme la constance des objectifs poursuivis au-delà des cadres territoriaux retenus pour l'action administrative.

41 La région s'est vue attribuer sur son territoire la responsabilité de définir des orientations en matière de développement économique, et pour ce faire, elle doit établir un schéma régional de développement économique, d'innovation et d'internationalisation. Il doit aussi faire l'objet d'une concertation avec les métropoles, les EPCI, et d'une discussion dans le cadre de la Conférence territoriale de l'action publique avec les organismes consulaires.

42 La mise en place d'un schéma régional d'aménagement et de développement durable du territoire associe les conseils départementaux et les intercommunalités à son élaboration.

43 Si la nouvelle planification confère aux régions une plus grande visibilité face à l'État et plus de légitimité, dans le même temps, l'application de la réforme va obliger les grandes régions à mettre de l'ordre dans les différents schémas et devrait mobiliser les collectivités infrarégionales et les habitants autour d'un projet commun.

La mise en place d'une planification prescriptive sera un levier d'action des régions et un outil de renforcement de l'échelon régional.

45 Par contre, la clarification des compétences risque de rester un vœu pieu... Le déséquilibre persiste entre l'État qui possède un socle de compétences dites « régaliennes » et les collectivités territoriales qui sont plurielles. Il est vrai que dès 1982 a été introduite la notion de «bloc de compétences " mais la répartition des compétences n'a pas suivi la logique des blocs. Et les lois intervenues depuis, extrêmement nombreuses, ont modifié directement tel point de la répartition des compétences ou ont eu une incidence sur leur répartition. La complexité de la réalité rend illusoire l'établissement d'un tel «bloc». Et l'État est demeuré infiniment plus puissant que chacune des collectivités territoriales.

46 Loin d'être nouvelle, la recherche de la clarification des compétences a fait l'objet de plusieurs lois. Celle du 16 décembre 2010 a cherché à supprimer la clause de compétence générale des départements et des régions. Mais des atténuations et des exceptions à cette spécialisation de compétences entre collectivités ont rendu caduque l'application de la loi. Les différents domaines à attribuer font l'objet de discussions et d'hésitations même si des compétences privilégiées semblent plus faciles à attribuer au département qu'à la région.

47 - Le département est reconnu pour assurer une mission de solidarité et de péréquation de la gestion des services d'aide sociale. L'ensemble des questions légales d'aide sociale lui fut très tôt confirmé.

48 - La région s'est vue attribuer des compétences limitées au domaine économique, social et culturel. Elle est une sorte d'animateur de la vie économique et sociale. En exerçant un rôle de réflexion et d'impulsion en matière d'aménagement du territoire et plus généralement de développement économique, la région s'est affirmée comme un échelon incontournable lors de la signature des contrats de plan.

49 Cependant dans d'autres domaines, notamment en matière d'éducation, de culture et de tourisme, le partage entre département et région donne lieu à concurrence entre 
l'État, la région, le département, la commune ; chaque collectivité étant très attachée à sa liberté de définition d'une politique qui lui est propre.

La réorganisation des compétences non accompagnée d'une disposition sur l'allocation des ressources et de la fiscalité fait peser un facteur d'incertitude.

51 L'inachèvement de la réforme et les incertitudes qu'elle laisse subsister font dire à Alain Rousset, président de l'Association des Régions de France, « qu'il s'agit plus d'une départementalisation des régions que d'une régionalisation des départements ».

Illustration 2 -Carte des chefs-lieux des nouvelles régions françaises

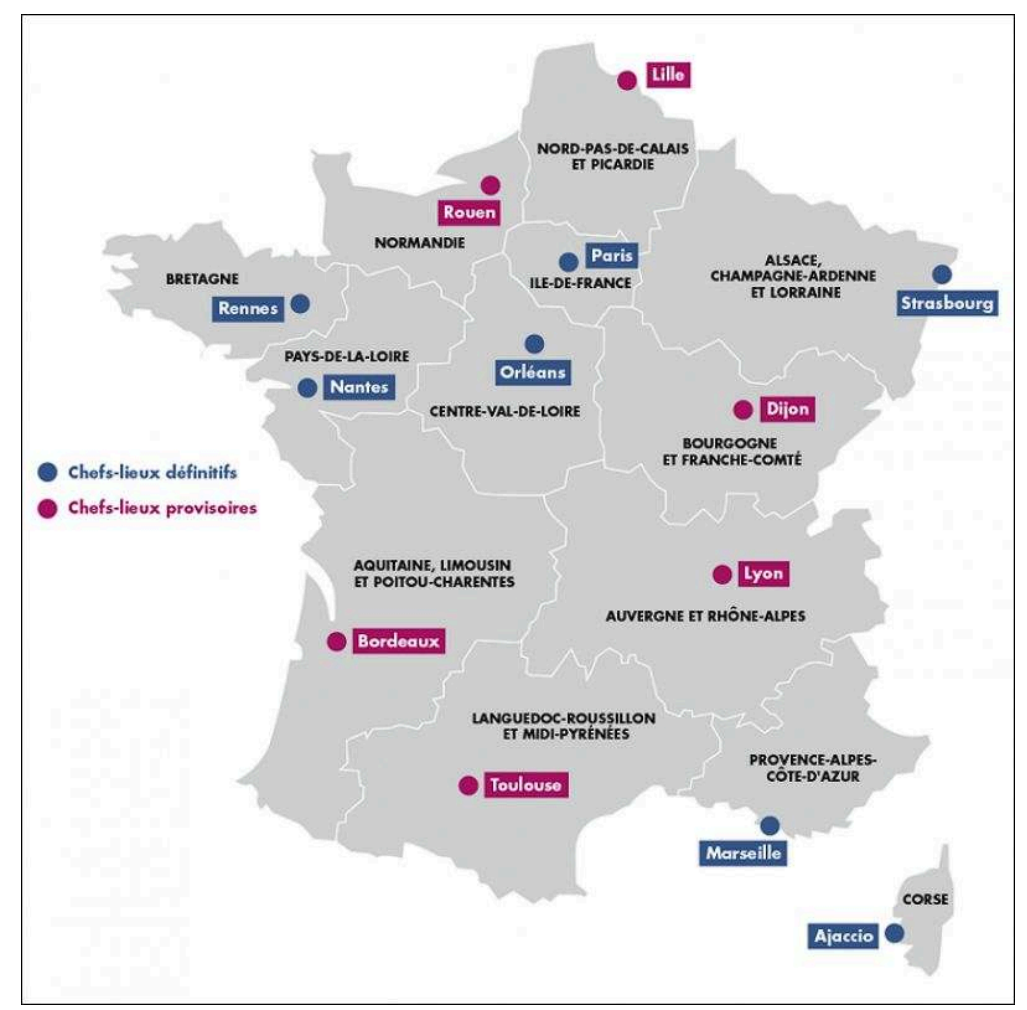

Le choix des chefs-lieux sera définitivement fixé par décret en Conseil d'État avant le 1er juillet 2016.

Source : Ministère de l'Intérieur

53 Si depuis les années 1960, le couple régions-villes a été perçu comme l'instrument de la modernisation du territoire, les villes devenues des pôles de développement économiques ont pu se substituer à la capitale régionale: "le couple région-réseau métropolitain est aujourd'hui en mesure de s'affirmer » (D. Béhar, 2015).

La reconnaissance de la place des villes a été confortée par l'affectation d'un statut différencié attribué aux métropoles par la loi du 27 janvier 2014. La loi acte le transfert de compétences de base et ouvre la possibilité «de compétences déléguées ou transférées à la métropole par l'État ». La reconnaissance juridique des métropoles dans la loi MAPTAM confirme leur rôle d'acteur économique à l'échelle régionale même si persiste entre métropoles et régions une compétition pour assurer le pilotage des stratégies d'innovation et de développement. La création de grandes régions s'est appuyée sur la métropolisation des territoires en sorte que l'élargissement du périmètre de chacune d'elles dispose au moins d'une métropole même si d'une 
métropole à l'autre le dynamisme économique et l'attractivité peuvent varier considérablement et même si les limites des pôles métropolitains sont parfois avant tout politiques.

Malgré l'émergence des métropoles comme acteurs économiques centraux, des calculs de valeur ajoutée en 2013-2014 pour chaque métropole à l'échelle de la région laissent apparaître dans le périmètre des nouvelles grandes régions un affaiblissement sensible de leur poids. Le meilleur exemple est fourni par Strasbourg, capitale de la région Alsace, pesant $40 \%$ de la richesse de la région et qui dans le Grand Est ne pèsera pas même $20 \%$.

À périmètre constant, les choses bougeront peu.

On ne peut présager des effets du redécoupage régional. Même si des économies sont attendues, elles risquent d'être largement contrebalancées par des frais de fusion. En outre les stratégies adoptées par les nouvelles gouvernances régionales, la redistribution des services sans oublier l'environnement économique national et international laissent planer de nombreuses incertitudes.

La création des métropoles a certes connu une accélération lors de la promulgation de la loi MAPTAM. Si la loi cherche à développer une logique de standardisation, force est de constater que l'existence de grandes métropoles, Paris en tête suivi de Lyon et Marseille, se singularise dans la répartition des rôles entre région, département et même territoires voisins.

59 La région capitale reste une «affaire d'État ». Le primat du politique et du niveau étatique en France a donné naissance à une articulation particulière entre la région capitale et les autres. Paris capitale doit réussir à composer avec l'État et la région. La région Ile-de-France compte 12,5 millions d'habitants et représente $31 \%$ du PNB. Seuls 8 euros sur 100 de dépenses sont imputables à la politique financière de la région, tout le reste venant de l'État rappelait Jean-Paul Huchon devant la Commission des lois le 24 juin 2014.

61 Le pays basque espagnol dispose d'un budget huit fois plus important sans que le poids de cette région soit comparable à celui de la région de l'Ile-de-France. Ainsi en termes de puissance, même la région capitale est loin d'autres régions européennes. Or la faiblesse des moyens non seulement persistera mais s'accroîtra.

62 La métropole du Grand Paris (MGP) est officiellement entrée en vigueur au $1^{\mathrm{er}}$ janvier 2016. Les enjeux de la mise en place du grand Paris et la répartition des compétences entre la nouvelle Métropole et le Conseil régional d'Ile- de-France alimentent de vives rivalités. Il est vrai que les enjeux sont nationaux et internationaux et l'État ne peut rester à l'écart de la construction métropolitaine.

63 Ce n'est qu'à partir de 2020 que la métropole parisienne sera dotée d'un statut spécifique avec 136 communes, 4 départements, la région et l'État. L'avenir permettra de mesurer ou non la plus grande lisibilité de la Métropole du Grand Paris.

64 À Marseille, l'impulsion de l'État a été décisive pour engager «un cycle de coconstruction métropolitaine». C'est en rapprochant les deux composantes que constituent le monde maritime dans l'histoire économique du port de Marseille et la haute technologie du moyen pays d'Aix que la métropole «Marseille Provence» a

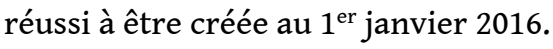



recentralisation repose sur une nécessaire collaboration entre la région, la métropole régionale qui détient le pouvoir en lien avec les acteurs économiques essentiels et les EPCI. s'est substituée au département du Rhône et en exerce les compétences sur le périmètre métropolitain. S'y est élaborée une gouvernance en convergence avec les milieux économiques. Elle offre un gouvernement intégré après être devenue une collectivité à statut particulier.

Hors ces grandes métropoles, chaque région dispose au moins d'une métropole ou d'un pôle urbain appelé à favoriser les interactions et la coopération entre les acteurs économiques et politiques. Il existe des régions sans métropole, la Corse par exemple.

\section{Conclusion}

La France continuera à présenter un entrelacs de collectivités nombreuses et dispendieuses. Si la réduction du nombre des régions était plus facile à conduire, pour autant les régions n'ont pas disqualifié les départements. Les communautés de communes (EPCI) font doublons en termes de périmètre avec les cantons (circonscriptions électorales départementales) ou les arrondissements (circonscriptions administratives de déconcentration des services de l'État). Les cadres administratifs prolifèrent. Ces maillages administratifs multiples contribuent au brouillage des politiques et ce d'autant plus qu'on retrouve les mêmes élus dans plusieurs structures gigognes voire concurrentes.

Si tout le monde admet que l'enchevêtrement des collectivités a fini par provoquer un émiettement des responsabilités et des politiques publiques, la simplification annoncée reste un vœu pieu.

Il eut fallu prendre le temps d'écouter et de consulter en impliquant les différents acteurs pour faire émerger les structurations soutenables et les recompositions souhaitables.

Dans son actuelle mouture, la réforme territoriale s'apparente plus à une recentralisation des politiques publiques à l'échelon de la région. Mais cette

\section{BIBLIOGRAPHIE}

Behar D., 2015. Changer les institutions ou changer les pratiques. Esprit, 2015/2, p. 85-95.

Behar D., 2015. Y a-t-il une bonne échelle locale ?, Esprit, 2015/2, p. 96-108.

Behar D., 2014. Paris, Lyon, Marseille : la gouvernance métropolitaine entre standardisation et différenciation. Cinquièmes journées « Grand Paris » de l'Ecole d'urbanisme de Paris, 20-21 mars 2014. Metropolitiques.eu 2014. http://www.metropolitiques.eu/Paris-Lyon-Marseille-la.html 
Dumont G.-F., 2012. Diagnostic et gouvernance des territoires, concepts, méthode, application. Armand Colin.

Estebe P., 2015. La décentralisation? Tous contre ! Esprit, 2015/2, p. 74-84.

Giblin B., 2015. L'obsession du local : une exception française ? Esprit, 2015/2, p. 64-73.

Giraut F., 2002. L'exception territoriale française sous influence européenne ? L'Information géographique, juin 2002, p. 133-161.

Jean Y., Vanier M., 2008. La France. Aménagement du territoire. Armand Colin.

Nemery J.-C., 2015. Quelle organisation pour les grandes régions en France et en Europe ? L'Harmattan.

Piercy P., 2014. La France. Le fait régional. Hachette, 5e édition.

Reghezza-Zitt M., 2011. La France dans ses territoires. SEDES.

Subra P., 2007. Géopolitique de l'aménagement du territoire. Armand Colin.

Vanier M., 2002. Recomposition territoriale française : la voie française. L'information

géographique, p. 99-112.

Vanier M., 2008. Le pouvoir des territoires. Essai sur l'interterritorialité. Economica.

\section{Rapports du Sénat}

La révolution de l'intercommunalité, 6 février 1992 - 6 février 2012, vingt ans de communautés de communes, bilan et perspectives, rapport n³79, Commission des lois 2011-2012.

Les préfectures à l'heure de la réorganisation de l'administration territoriale de l'État, RéATE, rapport d'information $n^{\circ} 77$, Commission des finances, 2013-2014.

Délimitation des Régions, élections régionales et départementales et modification du calendrier électoral, rapport $n^{\circ} 658$, Commission spéciale, 2013-2014.

\section{NOTES}

1. Au $1^{\mathrm{er}}$ janvier 2016, on recense 2062 établissements publics de coopération intercommunale (EPCI) à fiscalité propre sur le territoire français. Parmi ceux-ci, on dénombre 13 métropoles, 11 communautés urbaines, 196 communautés d'agglomération et 1842 communautés de communes. Le nombre de communes isolées s'élève à 27 sur le territoire national. (http:// www.collectivites-locales.gouv.fr/cartographie-des-epci-a-fiscalite-propre)

2. Cité dans le Rapport du Sénat, Commission spéciale $n^{\circ}$ 658, p 156.

\section{RÉSUMÉS}

À chaque année sa réforme : en 2014, la nouvelle carte administrative française ramène de 22 à 13 le nombre des régions. L'émergence de la région devenue une collectivité territoriale de plein exercice fut lente et consacrée seulement en 1982. Depuis quelques années, plusieurs rapports ont proposé la réduction du nombre des régions et une redéfinition des compétences des conseils 
départementaux et régionaux.

L'objet principal de la réforme visait à "doter les régions d'une taille critique » pour leur permettre d' " exercer les compétences stratégiques qui leur sont attribuées et de réaliser des gains d'efficience ».

Or la région n'est toujours pas consacrée comme chef de file et les conseils départementaux sont plus que jamais inscrits dans le paysage institutionnel aux côtés des intercommunalités de plus en plus larges. L'objectif d'économies ne pourra être obtenu que par le renforcement de ces intercommunalités, tant les dotations du gouvernement aux collectivités ont été rabotées. Et si les fusions ont été privilégiées, l'effet de taille est loin d'avoir été appliqué.

La réforme paraît être une occasion manquée ...

In 2014 the new administrative map of France reduced the number of regions from 22 to 13 . The emergence of the region as a territorial collectivity with specific responsibilities was slow and only became operational in 1982. In recent years, several reports have proposed a reduction in the number of regions and a redefinition of the responsibilities of departmental councils and of regional councils. The main objective of the reform sought to 'give the regions a critical size' to enable them 'to exercise the strategic responsibilities attributed to them and to achieve efficiency gains'. Alongside the regions, the departmental councils are inscribed into the institutional landscape, together with larger and larger intercommunal units (intercommunalités). The objective of making savings could only be achieved by reinforcing intercommunal units, and hence governmental responsibilities of other territorial collectivities have been planed down. Preference has been given to mergers, but the issue of scale has not been applied evenly. The reform appears to be a missed opportunity.

INDEX

Thèmes : Sur le Champ - Sur le Terrain

\section{AUTEUR}

\section{ÉLISABETH BONNET-PINEAU}

Elisabeth Bonnet Pineau, danieloster@wanadoo.fr, est géographe et consultante. 